\title{
Haplotype association analysis of North American Rheumatoid Arthritis Consortium data using a generalized linear model with regularization Wei Guo, Chin-yuan Liang and Shili Lin*
}

Address: Department of Statistics, The Ohio State University, Columbus, Ohio 43210 USA

E-mail: Wei Guo - guowei@stat.osu.edu; Chin-yuan Liang - liang.88@osu.edu; Shili Lin* - shili@stat.osu.edu

${ }^{*}$ Corresponding author

from Genetic Analysis Workshop 16

St Louis, MO, USA 17-20 September 2009

Published: 15 December 2009

BMC Proceedings 2009, 3(Suppl 7):S32 doi: 10.1186/1753-6561-3-S7-S32

This article is available from: http://www.biomedcentral.com//753-656I/3/S7/S32

(C) 2009 Guo et al; licensee BioMed Central Ltd.

This is an open access article distributed under the terms of the Creative Commons Attribution License (http://creativecommons.org/licenses/by/2.0), which permits unrestricted use, distribution, and reproduction in any medium, provided the original work is properly cited.

\begin{abstract}
The Genetic Analysis Workshop 16 rheumatoid arthritis data include a set of 868 cases and II 94 controls genotyped at 545,080 single-nucleotide polymorphisms (SNPs) from the Illumina $550 \mathrm{k}$ chip. We focus on investigating chromosomes 6 and 18, which have 35,574 and 16,450 SNPs, respectively. Association studies, including single SNP and haplotype-based analyses, were applied to the data on those two chromosomes. Specifically, we conducted a generalized linear model with regularization (rGLM) approach for detecting disease-haplotype association using unphased SNP data. A total of 444 and 43 four-SNP tests were found to be significant at the Bonferroni corrected $5 \%$ significance level on chromosome 6 and 18 , respectively.
\end{abstract}

\section{Background}

Genetic Analysis Workshop (GAW) 16 Problem 1 involves studies designed to investigate genetic risk factors for rheumatoid arthritis (RA). The data are the initial batch of whole-genome scans for the North American Rheumatoid Arthritis Consortium (NARAC) cases $\left(\mathrm{n}_{1}=868\right)$ and controls $\left(\mathrm{n}_{2}=1194\right)$. The HLA region on $6 \mathrm{p} 21$ has been implicated by numerous studies and there is consistent evidence that the DR alleles contribute to disease risk [1]. The region on chromosome $18 \mathrm{q}$ has also shown evidence for linkage to RA in U.S. and French linkage scans [2,3]. Therefore, we focused our association study on these two chromosomes.
Recent advances in molecular technology lead to the availability of a large number of SNPs, and there are increasing interest in association studies involving haplotypes defined by several closely linked SNPs. Haplotype association studies are being employed more and more to investigate associations for complex diseases [4]. The generalized linear model (GLM) is a flexible framework that allows for the incorporation of environment factors and interactions between covariates, in which a logistic regression model can be used for binary traits. When rare haplotypes are present, however, the standard log-likelihood approach for GLM could lead to large standard errors for the coefficients of such haplotypes. In fact, the expectation maximization (EM) 
algorithm, usually employed for estimating such parameters, might not converge at all. Moreover, it would lead to a large degrees of freedom in the haplotype test, and therefore reduced power when there is a large number of haplotypes in an association analysis. On the other hand, GLM with regularization (rGLM) can effectively combat these problems, and in particular, it is applicable to the common disease/rare variant scenario [5].

\section{Methods}

\section{Data checking}

As a quality control measure, we tested for HardyWeinberg equilibrium (HWE) in the controls using an exact test. There are 156 and 55 SNPs with HWE $p$-values less than $1.4 \times 10^{-6}$ and $3 \times 10^{-6}$ on chromosome 6 and 18 , respectively, which are significant $(p>0.05)$ after Bonferroni correction. Moreover, there are 106 and 59 SNPs with monomorphism. We also checked for SNPs with a large amount of missing data, but none of the SNPs were removed based on the criterion of at least $50 \%$ missing rate, which was chosen to keep SNPs with a reasonable amount of data in the preliminary step. Thus, a total of 262 and 114 on chromosomes 6 and 18, respectively, were removed either due to the lack of polymorphisms or significant deviations from HWE in the controls. All 2062 samples were used.

\section{rGLM}

To deal with the problems of large standard errors, nonconvergence, and reduced power associated with standard GLM likelihood approach, we adopted a statistical learning method that effectively shrinks the coefficients of unassociated haplotypes and reduces the variance of the estimated regression coefficients. One frequently used method for doing this is the use of the LASSO penalty, which shrinks the coefficients of unassociated variables to zeros [6]. This is implemented in the rGLM software [5], which assumes HWE and was used in this study.

rGLM applies the LASSO penalty to a logistic regression model on unphased genotype data. In a case-control study design, the complete data log-likelihood function for individual $i$ can be expressed as follows:

$$
l_{c i}(\theta)=\log \left[\operatorname{Pr}\left(y_{i} \mid X_{i}, \beta\right) \operatorname{Pr}\left(X_{i} \mid \gamma\right)\right],
$$

where $y_{i}$ and $X_{i}$ (missing) denote the trait value and haplotype of individual $i$, respectively, and $\beta$ and $\gamma$ are the logistic regression coefficients and haplotype frequency parameters. Using the LASSO penalty, the complete penalized likelihood function is

$$
l_{c}^{*}(\theta)=\sum_{i} l_{c i}(\theta)-\lambda \sum_{r-1}^{m-1}\left|\beta_{r}\right|,
$$

where $\lambda$ is the tuning parameter and $m$ is the number of haplotypes. This likelihood function can be maximized by the EM algorithm. To determine the tuning parameter $\lambda$, it makes use of a recent result in Zou et al. [7], which shows that the number of non-zero coefficients in a LASSO regression is an unbiased estimate of the degrees of freedom.

\section{Other analyses}

As a preliminary genome scan measure, single-SNP tests were carried out using a genotype-based Fisher's exact test. In addition to the rGLM approach, hapassoc [8] was also employed to test for association on haplotypes as a standard GLM likelihood approach, in which an EM algorithm was used to infer the haplotypes and haplotype effects simultaneously.

\section{Results}

For each single SNP on chromosome 6 and 18, an exact test was carried out based on genotype counts; the $p$-values are shown in Figure 1. There are 424 and 16 statistically significant SNP associations at the Bonferroni corrected level of 5\% for chromosome 6 and 18, respectively. It is interesting to note that on chromosome 6, except for 29 SNPs, all the remaining 395 are distributed in a small region, from position 29463092 (7448 ${ }^{\text {th }}$ SNP: rs238869) to position 33955055 (9113th SNP: rs10947463) (Figure 1, left panel), which covered the HLA-DRB1 allele and most of the HLA region on 6p21. On chromosome 18, 16 significant SNPs were found (Figure 1, right panel), which include SNPs that overlaps with those found in previous studies $[2,3]$.

Because single-SNP tests may be less powerful than haplotype-based tests in many situations, we carried out a two-step haplotype analysis. To reduce the computational demand for genome-wide analysis, in the first step we performed a logistic regression with the LASSO penalty using glmpath [9] using all SNPs assuming an additive model between SNPs and a co-dominant model for each SNP. The missing values were replaced by the most frequent genotypes for the corresponding SNPs. As a result, there were 986 and 249 'tag' SNPs selected on chromosome 6 and 18, respectively. We note that these so called 'tag' SNPs are not the conventional kind that can be considered as the 'proxy' for those not selected. Instead, they are tagged due to their likely association with the disease.

In the second step, based on the selected 'tag' SNPs, a four-SNP sliding window was taken to implement the 

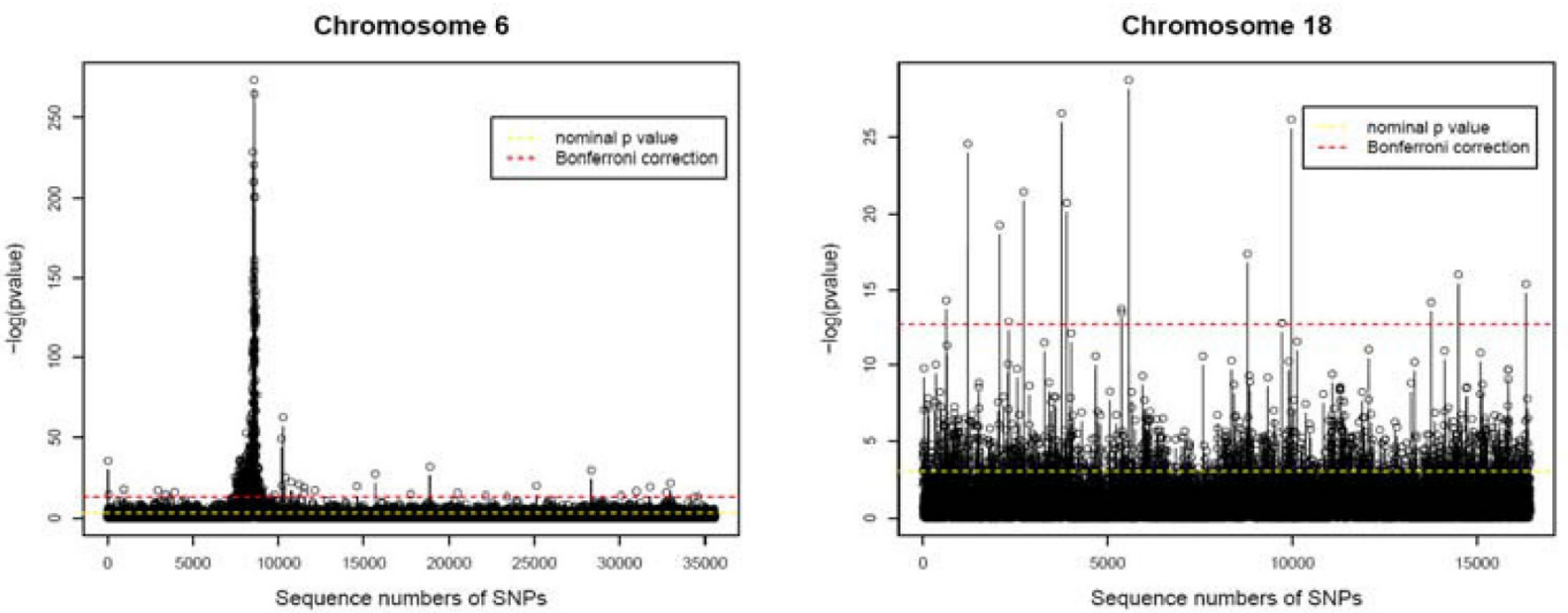

Figure I

Single SNP association. The -log p-values for a whole genome analysis by genotype-based exact tests on chromosome 6 and 18. The statistically significant associations at nominal level (yellow line) and at Bonferroni corrected level (red line) are also indicated.

haplotype approaches, rGLM and hapassoc. Due to the existence of rare haplotypes, $64 \%$ (out of the total of 1229 four-SNP tests on both chromosomes) of the tests using hapassoc did not converge, whereas rGLM did not encounter such a problem. As shown in Figure 2, for those tests that hapassoc was able to run, analyses yielded 309 and 16 significant results for chromosome 6 and 18, respectively. On the other hand, using the number of nonzero coefficients as an estimate of the degrees of freedom [7], the rGLM gave, for chromosomes 6 and 18, respectively, 444 and 43 significance test results. Indeed, rGLM was able to identify additional significant tests through alleviating the problem of nonconvergence. For example, on chromosome 18, all of the 16 significant results identified by hapassoc were included in those found by rGLM. Furthermore, rGLM uncovered 27 additional ones from among the 64\% of

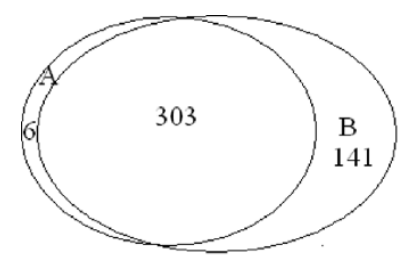

Chromosome 6

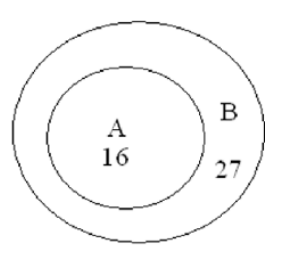

Chromosome 18
Figure 2

rGLM vs. hapassoc. The Venn diagrams for the results from rGLM and hapassoc. A, significant hapassoc tests; B, significant rGLM tests. Left panel and right panel are for chromosomes 6 and 18, respectively. tests that hapassoc failed to converge. We plotted the minimum frequencies of the significant four-SNP windows (Figure 3), which shows that the distribution of haplotype frequencies among the significant results identified by rGLM indeed contains rarer haplotypes than the distribution representing the frequencies of those identified by hapassoc, reaffirming the value of rGLM for detecting rare variants.

\section{Discussion}

We focused on scanning the SNPs on chromosomes 6 and 18 in our analysis based on evidence from prior studies. For chromosome 6, both single-SNP and haplotype-based approaches identified numerous associated SNPs/haplotypes around the HLA region, solidifying the importance of the HLA region for autoimmune

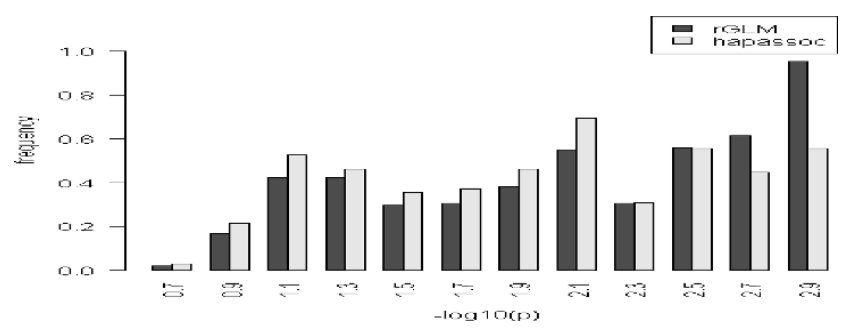

Figure 3

Frequency distributions of haplotypes. Histograms of minimum haplotype frequencies (after negative logarithm transformation) of the significant 4-SNP tests identified by rGLM and hapassoc. X-axis denotes the center of each bar and the bar width is 0.2 . 
diseases and confirming results from previous studies. Despite many common findings, each of the haplotypebased approaches identified more than 100 four-SNP windows that do not contain any of the significant SNPs selected by single-SNP analysis. This may be explained by the increased power of haplotype analysis, but further investigation is needed.

It is challenging to run whole-genome haplotype-based analysis with only phased-unknown SNP data. To reduce dimensionality, one of the most frequently employed approaches is to find tagging SNPs before embarking on a haplotype-based analysis. We attempted to use the haploview software for such as task. However, the amount of SNP reduction was not sufficient for the subsequent haplotype analysis to be practically feasible - less than 20\% of the SNPs were excluded on each chromosome using haploview. On the other hand, the penalized regression approach as described earlier was able to accomplish this task, leading to the identification of about 3\% of SNPs as 'tags'. This remarkable reduction makes our haplotype-based analysis, as well as other computationally intensive approaches for genome-wide studies, possible. However, the loss of information needs to be investigated further.

Using a penalized approach, rGLM shows a good power for detecting the effects of rare haplotypes [5]. Compared to the usual unpenalized GLM, rGLM is powerful and does not encounter the problem of non-convergence. However, the permutation procedure as proposed in Guo and Lin [5] can be too computationally intensive for obtaining $p$-values for studies on a genome-wide scale. Instead, we only obtained $p$-values by permutation and also by chi-square approximations (two different ways, one conservative and one liberal) on a selected subset to gauge whether chi-square approximation will give reasonably good results in this application. We found that for SNP combinations that give small $p$-values (say uncorrected $p<0.01$ ), all three methods lead to the same conclusion. Because our interest is in identifying significant haplotypes, we feel that our approximation method for computing the $p$-value is reasonable. However, further research on the appropriateness of such an approximation procedure and whether this will lead to the same type I error rate for hapassoc and rGLM is warranted.

\section{List of abbreviations used}

EM: Expectation maximization; GAW: Genetic Analysis Workshop; GLM: Generalized linear model; HWE: Hardy-Weinberg equilibrium; NARAC: North American Rheumatoid Arthritis Consortium; RA: Rheumatoid arthritis; rGLM: Generalized linear model with regularization; SNP: Single-nucleotide polymorphism.

\section{Competing interests}

The authors declare that they have no competing interests.

\section{Authors' contributions}

WG and SL developed the methodology and devised the analysis scheme. WG carried out the analysis with the assistance of CL. GW and SL drafted the manuscript. All authors read and approved the final manuscript.

\section{Acknowledgements}

The Genetic Analysis Workshops are supported by NIH grant ROI GM03 I575 from the National Institute of General Medical Sciences. This research was supported in part by NIH grant ROI HG002657 and by a Biomedical Research and Technology Transfer grant from the State of Ohio Tech 05-062.

This article has been published as part of BMC Proceedings Volume 3 Supplement 7, 2009: Genetic Analysis Workshop 16. The full contents of the supplement are available online at http://www.biomedcentral.com/ $1753-656 \mid / 3$ ? issue $=$ S7.

\section{References}

I. Amos Cl, Chen WV, Seldin MF, Remmers E, Taylor KE, Criswell LA, Lee AT, Plenge RM, Kastner DL and Gregersen PK: Data for Genetic Analysis Workshop I6 Problem I, association analysis of rheumatoid arthritis data. BMC Proceedings 2009, 3 (Suppl 7):S2.

2. Jawaheer D, Seldin MF, Amos Cl, Chen WV, Shigeta R, Etzel C Damle A, Xiao X, Chen D, Lum RF, Monteiro J, Kern M, Criswell LA, Albani S, Nelson JL, Clegg DO, Pope R, Schroeder HW Jr, Bridges SL Jr, Pisetsky DS, Ward R, Kastner DL, Wilder RL, Pincus T, Callahan LF, Flemming D, Wener MH, Gregersen PK and North American Rheumatoid Arthritis Consortium: Screening the genome for rheumatoid arthritis susceptibility genes: a replication study and combined analysis of 512 multicase families. Arthritis Rheum 2003, 48:906-916.

3. Osorio Y, Fortéa J, Bukulmez H, Petit-Teixeira E, Michou L, Pierlot C, Cailleau-Moindrault S, Lemaire I, Lasbleiz S, Alibert O, Quillet P, Bardin T, Prum B, Olson JM and Cornélis F: Dense genome-wide linkage analysis of rheumatoid arthritis, including covariates. Arthritis Rheum 2004, 50:2757-2765.

4. Schaid D): Evaluating associations of haplotypes with traits. Genet Epidemiol 2004, 27:348-364.

5. Guo $W$ and Lin SL: Generalized linear modeling with regularization for detecting common disease rare haplotype association. Genet Epidemiol 2009, 33:308-316.

6. Tibshirani R: Regression shrinkage and selection via the lasso. J R Stat Soc Series B Stat Methodol 1996, 58:267-288.

7. Zou H, Hastie T and Tibshirani R: On the "degrees of freedom" of the lasso. Ann Stat 2007, 35:2173-2192.

8. Burkett K, Graham J and McNeney B: Hapassoc: Software for likelihood inference of trait associations with SNP haplotypes and other attributes. Stat Softw 2006, I6: I-19.

9. Park MY and Hastie T: LI regularization path algorithm for generalized linear models. J R Stat Soc Series B Stat Methodol 2007, 69:659-677. 